TRANSACTIONS OF THE

AMERICAN MATHEMATICAL SOCIETY

Volume 355 , Number 10, Pages 4187-4200

S 0002-9947(03)03348-8

Article electronically published on June 24, 2003

\title{
FIBRED KNOTS AND TWISTED ALEXANDER INVARIANTS
}

\author{
JAE CHOON CHA
}

\begin{abstract}
We study the twisted Alexander invariants of fibred knots. We establish necessary conditions on the twisted Alexander invariants for a knot to be fibred, and develop a practical method to compute the twisted Alexander invariants from the homotopy type of a monodromy. It is illustrated that the twisted Alexander invariants carry more information on fibredness than the classical Alexander invariants, even for knots with trivial Alexander polynomials.
\end{abstract}

\section{IntRoduction AND MAIN RESUlts}

Although the classical Alexander invariants of knots in the three space have been sucessfully used as the most fundamental tools in studying various problems, it is also well known that they are not sharp enough in many cases. One approach to overcome this limitation is to consider the twisted Alexander invariants, which were first defined in the works of Lin [13, Wada 16], and Jiang and Wang [8, and subsequently studied by several authors including Kitano [11] and Kirk and Livingston [9, 10].

A common but important question is how a particular geometric property is reflected on the twisted Alexander invariants, and, furthermore, whether it gives more information on the geometric property than the classical Alexander invariants. In the remarkable work of Kirk and Livingston [9], [10], they introduced a natural topological definition of the twisted Alexander polynomial, which turned out to be an important innovation for this purpose. For a surjection of the fundamental group $\pi$ of a complex $X$ onto $\mathbf{Z}$ and a representation of $\pi$ over a field $F$, they defined the twisted Alexander module to be a specific twisted homology group of $X$, which is a module over the principal ideal domain $F[\mathbf{Z}]$, and defined the twisted Alexander polynomial to be a polynomial representing the order of (the torsion part of) the twisted Alexander module. Some known results on the Alexander polynomial were extended to the twisted case. In particular, they obtained a knot slicing obstruction from the twisted Alexander polynomials associated to specific representations of fundamental groups of prime power fold cyclic branched covers, by relating them with the Casson-Gordon invariants, and illustrated that the obstruction detects nonslice knots with Alexander invariants of slice knots.

The aim of this paper is to study the twisted Alexander invariants of fibred knots along the same lines. We establish necessary conditions on the twisted Alexander invariants for a knot to be fibred, which can be viewed as a generalization of the

Received by the editors October 5, 2001 and, in revised form, February 15, 2003.

2000 Mathematics Subject Classification. Primary 57M25.

Key words and phrases. Fibred knots, twisted Alexander invariants.

(C)2003 American Mathematical Society 
well-known fact that the Alexander polynomial of a fibred knot is monic, i.e., the coefficients of the highest and lowest degree terms are units. For this purpose, we generalize the approach of Kirk and Livingston for a Noetherian unique factorization domain $R$ which is not necessarily a field. Roughly speaking, first we define the twisted Alexander ideal to be the elementary ideal of the twisted Alexander module, and then we define the twisted Alexander polynomial to be the greatest common divisor of specific generators of the elementary ideal. When $R$ is a field, the twisted Alexander polynomial of ours coincides with that of Kirk and Livingston if the former is nontrivial. (For details, see Section 2.)

Using the above terminologies, our main result is stated as follows. Suppose that every submodule of a free module of finite rank over the base domain $R$ is again free of finite rank. The main example to keep in mind is $R=\mathbf{Z}$.

Theorem 1.1. If $K$ is a fibred knot and $\rho$ is a representation of the fundamental group of a cyclic cover of $S^{3}$ branched along $K$ that factors through a finite group, then the twisted Alexander invariants associated to $\rho$ have the following properties:

(1) The twisted Alexander module is $R[\mathbf{Z}]$-torsion.

(2) The twisted Alexander ideal is a principal ideal generated by the twisted Alexander polynomial.

(3) The twisted Alexander polynomial is monic.

Note that if $R$ is a field, then the conclusions of Theorem 1.1 hold for any knot with a nontrivial twisted Alexander polynomial. This is the reason why we work with the twisted Alexander invariants over a domain $R$ which is not a field.

We also develop a practical computational method. In Section 3 we illustrate a simple algorithm computing the twisted Alexander invariants of a fibred knot from (the given representation and) the homotopy type of a monodromy on a fibre surface, which can be made easier to handle by describing it as a map of a graph, or equivalently as an automorphism of a free group. Theorem 1.1 is proved using this method.

The computational method itself can also be viewed as a generalization of the fact that the classical Alexander invariants can be computed from the homology type of a monodromy. In addition, in the case of knots that are "homologically" fibred but not homotopically, it explains why there is potential room for the twisted Alexander invariants to have more information than the classical ones. The latter part of this paper is devoted to exhibit that this is indeed true by examples. This is an interesting fact from the viewpoint of the study of the twisted Alexander invariants; although, it could be shown that our examples are not fibred by some other methods if one were interested in fibredness only. In Section 4 , we show that for any (possibly fibred) knot $K$ with a nontrivial Alexander polynomial, there exist infinitely many knots having the same classical Alexander invariants as $K$ but twisted Alexander invariants of nonfibred knots, that is, the conclusions of Theorem[1.1 are not satisfied. For this purpose, we investigate twisted Alexander invariants associated to "abelian" representations (whose images are abelian subgroups of $\mathrm{GL}_{n}$ ).

In a recent work of Cochran [3, a similar result has been proved using "higher order" Alexander modules. For knots with trivial Alexander polynomials, higher order Alexander modules have no more information than classical ones, as remarked in [3], and so do twisted Alexander invariants associated to abelian representations, as explained at the end of Section 4. However, we exhibit an interesting phenomenon that twisted Alexander invariants associated to nonabelian representations still 
have useful information on fibredness; in Section 5] we show that there are knots having trivial Alexander polynomials but twisted Alexander invariants of nonfibred knots.

\section{Twisted Alexander invariants}

We begin with definitions of the twisted Alexander invariants, which generalize the twisted Alexander polynomial defined in [9]. Throughout this section, we assume that $R$ is a Noetherian unique factorization domain. Let $X$ be a finite CW-complex and $\tilde{X}$ its universal covering space. $\pi_{1}(X)$ acts on the left of $\tilde{X}$ as the covering transformation group. Let $\epsilon: \pi_{1}(X) \rightarrow\langle s\rangle$ be a surjection, where $\langle s\rangle$ denotes the infinite cyclic group generated by $s$. We identify the group ring $R[\langle s\rangle]$ with the Laurent polynomial ring $R\left[s, s^{-1}\right]$ and denote it by $\Lambda$. Suppose that $\pi_{1}(X)$ acts on the right of a free $R$-module $V$ of finite rank via a representation $\pi_{1}(X) \rightarrow \mathrm{GL}(V)$. Then $\Lambda \otimes_{R} V$ becomes a $\Lambda-R\left[\pi_{1}(X)\right]$ bimodule under the actions given by $s^{k} \cdot\left(s^{n} \otimes v\right)=s^{n+k} \otimes v$ and $\left(s^{n} \otimes v\right) \cdot g=s^{n} \epsilon(g) \otimes v \cdot g$ for $v \in V$ and $g \in \pi_{1}(X)$. Let $C_{*}(\tilde{X} ; R)$ be the cellular chain complex of $\tilde{X}$ with coefficient $R$, which is a left $R\left[\pi_{1}(X)\right]$-module. The twisted cellular complex of $X$ with coefficient $\Lambda \otimes V$ is defined to be the following chain complex of left $\Lambda$-modules:

$$
C_{*}(X ; \Lambda \otimes V)=(\Lambda \otimes V) \otimes_{R\left[\pi_{1}(X)\right]} C_{*}(\tilde{X} ; R) .
$$

The twisted homology $H_{*}(X ; \Lambda \otimes V)$ is defined to be the homology of $C_{*}(X ; \Lambda \otimes V)$. We call $H_{1}(X ; \Lambda \otimes V)$ the twisted Alexander module. For notational convenience, we denote it by $A$.

Since $V$ is finitely generated, so is $A$ as a $\Lambda$-module. Since $R$ is Noetherian, so is $\Lambda$, and hence $A$ is a finitely presentable $\Lambda$-module. Choose a presentation of $A$, and let $n$ and $m$ be the numbers of generators and relations, respectively. Let $P$ be the $n \times m$ matrix associated to the presentation, i.e., the $(i, j)$-entry of $P$ is the coefficient of the $i$-th generator in the $j$-th relation. Let $D$ be the set of the determinants of all $n \times n$ submatrices obtained by removing $(m-n)$ columns from $P$. The ideal $\mathcal{A}$ in $\Lambda$ generated by $D$ is called the elementary ideal of $A$. It is known that the elementary ideal is an invariant of the $\Lambda$-module $A$, which is independent of the choice of $P$. For a proof, see [4, p.101]. A similar argument shows that the greatest common divisor $\Delta$ of elements of $D$ is also an invariant of $A$, which is well defined up to multiplication of $u s^{n}$ with $u$ a unit in $R$. (If $n>m$, $A=\{0\}$ and $\Delta=0$ by a convention.) We call $\mathcal{A}$ and $\Delta$ the twisted Alexander ideal and the twisted Alexander polynomial, respectively.

Remark 2.1. The elementary ideal of a module is contained in the annihilator ideal. In particular, elements of $\mathcal{A}$ annihilate $A$.

Remark 2.2. When $R$ is a field and $A$ is $\Lambda$-torsion, our definition is equivalent to that of Kirk and Livingston [9, §2]. In this case $\Lambda$ is a principal ideal domain, and by the classification theorem of finitely generated modules over $\Lambda, A$ is decomposed into a direct sum of cyclic modules $\bigoplus_{i} R\left[s, s^{-1}\right] /\left\langle d_{i}\right\rangle$, where $d_{i}$ is an element of $\Lambda$. Kirk and Livingston defined the twisted Alexander polynomial to be the product of all nonzero $d_{i}$. It is equal to the order of the torsion part of $A$. Since the diagonal matrix with diagonals $d_{i}$ is a presentation matrix of $A, \Delta$ is equal to the product of all $d_{i}$. This shows that the two definitions coincide if $\Delta \neq 0$, or equivalently $A$ is torsion. Furthermore, when $R$ is a field, $\mathcal{A}$ is the principal ideal $\langle\Delta\rangle$. In general, 
$\mathcal{A}$ is contained in $\langle\Delta\rangle$, however, the converse is not true if $R$ is not necessarily a field.

Remark 2.3. Using Fox's calculus, from a presentation of $\pi_{1}(X)$ one can compute the boundary map $C_{2}(X ; \Lambda \otimes V) \rightarrow C_{1}(X ; \Lambda \otimes V)$. Consequently, the twisted Alexander invariants can also be computed. In [9], this method was used as the main computational technique and was used to relate this topological version of twisted Alexander polynomial with Wada's invariants [16]. Because this method is not used in this paper, we do not proceed with further details.

Specifically, we define the twisted Alexander invariants of an oriented knot $K$ in $S^{3}$ as follows. The first homology of the exterior $E$, which is obtained by removing an open tubular neighborhood of $K$ from $S^{3}$, is an infinite cyclic group generated by an element $t$ such that $\operatorname{lk}(K, t)=+1$. Let $N$ be the $d$-fold cyclic cover of $E$. The image of the composition $\pi_{1}(N) \rightarrow \pi_{1}(E) \rightarrow H_{1}(E)=\langle t\rangle$ is the subgroup generated by $s=t^{d}$. Thus it induces a surjection $\epsilon: \pi_{1}(N) \rightarrow\langle s\rangle$ so that the twisted Alexander invariants of $N$ are defined for any representation of $\pi_{1}(N)$. In this paper, we will consider only representations that factor through the fundamental group of the $d$-fold cyclic cover $M$ of $S^{3}$ branched along $K$. View $N$ as a subspace of $M$, and let $i_{*}: \pi_{1}(N) \rightarrow \pi_{1}(M)$ be the homomorphism induced by the inclusion. For a representation $\rho$ of $\pi_{1}(M)$, we denote the twisted Alexander module, ideal and polynomial of $N$ associated to $\epsilon$ and $\rho i_{*}$ by $A_{K}^{\rho}, \mathcal{A}_{K}^{\rho}$, and $\Delta_{K}^{\rho}$, respectively.

Remark 2.4. The twisted Alexander invariants of the exterior $E$ associated to $\pi_{1}(E) \rightarrow H_{1}(E)=\langle t\rangle$ and a representation of $\pi_{1}(E)$ are also useful in studying knots. This version appears in some literature including [16, 11], 9], 10], where the last two concern our version (with field coefficients) as well.

\section{COMPUTATION FOR FIBRED KNOTS}

Let $K$ be a fibred knot, and let $M$ be the $d$-fold branched cover of $K$ as before. In this section, we are interested in a special case of representations that factor through finite groups; we assume that a given representation $\rho$ of $\pi_{1}(M)$ is decomposed as

$$
\rho: \pi_{1}(M) \stackrel{\phi}{\rightarrow} G \rightarrow \mathrm{GL}(V)
$$

where $\phi$ is a homomorphism into a finite group $G$ and $G \rightarrow \mathrm{GL}(V)$ is a representation in a free $R$-module $V$ of finite rank. Without any loss of generality, we may assume that $\phi$ is surjective. In addition, we assume that $R$ has the property that every submodule of a free $R$-module of finite rank is again free of finite rank. For example, the ring $\mathbf{Z}$ has this property.

We will compute the twisted Alexander invariants of $K$ associated to $\rho$ from a monodromy of $K$. Let $F$ be a fibre surface of $K$ and $h: F \rightarrow F$ a monodromy such that

$$
E=\mathbf{R} \times F /(r, x) \sim(r+1, h(x)), \quad r \in \mathbf{R}, x \in F,
$$

is the exterior of $K$ and $\{0\} \times \partial F$ represents a preferred longitude of $K$. Then the $d$-fold cyclic cover $N$ of the exterior is given by

$$
N=\mathbf{R} \times F /(r, x) \sim\left(r+d, h^{d}(x)\right), \quad r \in \mathbf{R}, x \in F .
$$

The preferred generator of the covering transformation group acts on $N$ by $[r, x] \mapsto$ $[r+1, h(x)]$. We note that any fibre surface $F$ is connected, and so is $N$. 
First, we need to compute $\pi_{1}(N)$ and $\pi_{1}(M)$. Since $N$ can be viewed as a quotient space of $[0, d] \times F$ under an obvious identification, $\pi_{1}(N)$ is expressed as an HNN-extension of $\pi_{1}(F)$. An explicit description is as follows. For convenience, we may assume that $h$ fixes a basepoint $*$ on $F$ by isotoping $h$. Then $\pi_{1}(N)$ is presented as

$$
\left.\pi_{1}(N)=\left\langle s, \pi_{1}(F)\right| s z s^{-1}=h_{*}^{d}(z) \text { for } z \in \pi_{1}(F)\right\rangle,
$$

where $h_{*}$ is the endomorphism on $\pi_{1}(F)$ induced by $h$. The map $\epsilon$ defined in the previous section is equal to the surjection $\pi_{1}(N) \rightarrow \pi_{1}(N) / \pi_{1}(F)=\langle s\rangle$. Since $\pi_{1}(M)$ is obtained from $\pi_{1}(N)$ by killing $s, \pi_{1}(M)$ is isomorphic to the quotient group of $\pi_{1}(F)$ modulo the normal subgroup generated by $\left\{z^{-1} h_{*}^{d}(z) \mid z \in \pi_{1}(F)\right\}$.

Let $\tilde{F}$ be the connected regular covering of $F$ associated to the composition

$$
\alpha: \pi_{1}(F) \rightarrow \pi_{1}(M) \stackrel{\phi}{\rightarrow} G
$$

that is, the kernel of $\alpha$ is equal to the image of the injection $\pi_{1}(\tilde{F}) \rightarrow \pi_{1}(F)$ induced by the covering projection. $G$ acts on $\tilde{F}$ as the covering transformation group. We need the following lemma, which is an easy exercise in the covering space theory. Since the author has not found a proof in the literature, we give a proof for completeness.

Lemma 3.1. Suppose that $p:\left(\tilde{X}, \tilde{x}_{0}\right) \rightarrow\left(X, x_{0}\right)$ is a regular covering projection, $\tilde{X}$ and $X$ are connected and locally path connected, and $f:\left(X, x_{0}\right) \rightarrow\left(X, x_{0}\right)$ is a map. If $z^{-1} f_{*}(z) \in p_{*} \pi_{1}(\tilde{X})$ for all $z \in \pi_{1}(X)$, then $f$ is lifted to a map $\tilde{f}: \tilde{X} \rightarrow \tilde{X}$ which commutes with the action of covering transformations, i.e., $\tilde{f} \tau=\tau \tilde{f}$ for any covering transformation $\tau$.

Proof. Choose $\tilde{x}_{0} \in p^{-1}\left(x_{0}\right)$. By the hypothesis $f_{*}(z) \in z \cdot p_{*} \pi_{1}(\tilde{X}), f_{*} p_{*} \pi_{1}(\tilde{X}) \subset$ $p_{*} \pi_{1}(\tilde{X})$. Thus there is a lift $\tilde{f}$ of $f$ fixing $\tilde{x}_{0}$ by the lifting criterion. Let $\tau$ be a covering transformation on $\tilde{X}$. Since $\tilde{f} \tau$ and $\tau \tilde{f}$ are lifts of the same map $f p$, it suffices to show that $\tilde{f} \tau\left(\tilde{x}_{0}\right)$ and $\tau \tilde{f}\left(\tilde{x}_{0}\right)=\tau\left(\tilde{x}_{0}\right)$ coincide, by the uniqueness of a lift. Let $\tilde{\delta}$ be a path from $\tilde{x}_{0}$ to $\tau\left(\tilde{x}_{0}\right)$. Then $\tilde{f} \tau\left(\tilde{x}_{0}\right)$ is the endpoint of $\tilde{f} \tilde{\delta}$. Since $\left[p \tilde{\delta}^{-1} \cdot p \tilde{f} \tilde{\delta}\right]=\left[p \tilde{\delta}^{-1} \cdot f p \tilde{\delta}\right]=[p \delta]^{-1} f_{*}([p \delta]) \in p_{*} \pi_{1}(\tilde{X})$, the endpoints of $\tilde{f} \tilde{\delta}$ and $\tilde{\delta}$ are the same.

In our case, $z^{-1} h_{*}^{d}(z)$ is contained in the kernel of $\alpha$ for any $z \in \pi_{1}(F)$. Thus by the lemma, the homeomorphism $h^{d}: F \rightarrow F$ is lifted to a homeomorphism $\tilde{h}^{d}: \tilde{F} \rightarrow \tilde{F}$ which commutes with the action of $G$. Now $\langle s\rangle \oplus G$ acts on $\mathbf{R} \times \tilde{F}$ by $\left(s^{n}, g\right) \cdot(r, w)=\left(r+n d,\left(\tilde{h}^{d}\right)^{n}(g \cdot w)\right)$. It is easily checked that the orbit space is $N$, and the projection $\mathbf{R} \times \tilde{F} \rightarrow N$ is a covering projection with covering transformation group $\langle s\rangle \oplus G$. This shows that $\mathbf{R} \times \tilde{F}$ is the regular covering of $N$ associated to the homomorphism $\epsilon \oplus \phi i_{*}: \pi_{1}(N) \rightarrow\langle s\rangle \oplus G$, where $i_{*}: \pi_{1}(N) \rightarrow \pi_{1}(M)$ is the map induced by the inclusion.

Recall that the twisted Alexander module $A_{K}^{\rho}$ is defined to be the twisted homology group $H_{1}(N ; \Lambda \otimes V)$ where $\Lambda=R[\langle s\rangle]$. It is equal to the first homology of the chain complex

$$
(\Lambda \otimes V) \otimes_{R[\langle s\rangle \oplus G]} C_{*}(\mathbf{R} \times \tilde{F} ; R) \cong V \otimes_{R[G]} C_{*}(\mathbf{R} \times \tilde{F} ; R) .
$$

As an $R$-module, it can be viewed as the twisted homology group $H_{1}(\mathbf{R} \times F ; V)=$ $H_{1}(F ; V)$. Since $F$ is a connected surface with nonempty boundary, $F$ has the 
homotopy type of a graph (1-complex) with one vertex. Therefore, $H_{1}(F ; V)$ can be computed from a chain complex

$$
\cdots \rightarrow 0 \rightarrow V \otimes_{R[G]} R[G]^{n} \stackrel{\partial_{1}}{\longrightarrow} V \otimes_{R[G]} R[G] \rightarrow 0
$$

where $n$ is the number of edges of the graph. Since $H_{1}(F ; V)=\operatorname{Ker}\left(\partial_{1}\right)$ is a submodule of $V \otimes_{R[G]} R[G]^{n}=V^{n}$, it is a free $R$-module of finite rank.

The action of $s$ on $H_{1}(F ; V)=H_{1}\left(V \otimes_{R[G]} C_{*}(\tilde{F})\right)$ is given by the homomorphism induced by $\tilde{h}^{d}: \tilde{F} \rightarrow \tilde{F}$. Let $H$ be a matrix associated to the induced map by choosing an $R$-basis of $H_{1}(F ; V)$. Then obviously $s I-H$ is a presentation matrix of $A_{K}^{\rho}$, as a $\Lambda$-module, where $I$ is the identity matrix. Since it is a square matrix, $\mathcal{A}_{K}^{\rho}$ is the principal ideal generated by $\Delta_{K}^{\rho}(s)=\operatorname{det}(s I-H)$.

We summarize the above discussion as a theorem.

Theorem 3.2. $\quad$ (1) $A_{K}^{\rho}$ is presented by the matrix $s I-H$, as a $\Lambda$-module.

(2) $\mathcal{A}_{K}^{\rho}$ is the principal ideal generated by $\Delta_{K}^{\rho}(s)$.

(3) $\Delta_{K}^{\rho}(s)=\operatorname{det}(s I-H)$.

Now Theorem 1.1 is easily proved.

Proof of Theorem [1.1. By Remark 2.1 $A_{K}^{\rho}$ is annihilated by $\Delta_{K}^{\rho}(s)$, and so the first conclusion follows. The second conclusion is no more than Theorem 3.2 (2). Finally, the coefficient of the highest term of $\Delta_{K}^{\rho}(s)$ is equal to $\operatorname{det}(I)=1$, and the constant term of $\Delta_{K}^{\rho}(s)$ is $\operatorname{det}(H)$, which is a unit in $R$ since $\tilde{h}^{d}$ is a homeomorphism on $\tilde{F}$. This completes the proof.

The following consequence of our discussion will be useful later.

Corollary 3.3. $A_{K}^{\rho}$ is annihilated by a monic polynomial.

Remark 3.4. If $V=R[G]$ and $G \rightarrow \mathrm{GL}(V)$ is the regular representation, all results of this section are true without the assumption that every submodule of a free $R$ module of finite rank is free of finite rank. For, in this case, the twisted homology $H_{1}(F ; V)$ is equal to $H_{1}(\tilde{F} ; R)$, and hence it is always a free $R$-module of finite rank since $\tilde{F}$ is a surface without closed components.

We finish this section with an example that illustrates our computational method for twisted Alexander invariants of fibred knots.

Example. Let $K$ be the trefoil knot. There is a well-known fibre structure of the exterior of $K$; e.g., see 15 for a detailed description. We need only the following fact: a monodromy of $K$ has the homotopy type of a map $h$ on a graph $B$ with one vertex and two oriented edges $x$ and $y$, which is defined by $h(x)=y^{-1}$ and $h(y)=x y$.

Then the fundamental group of the double branched cover $M$ of $K$ is given by

$$
\pi_{1}(M)=\left\langle x, y \mid x=h^{2}(x)=y^{-1} x^{-1}, y=h^{2}(y)=y^{-1} x y\right\rangle .
$$

By simplifying relations, $\pi_{1}(M)$ is a cyclic group of order 3 generated by $x=y$. Let $\rho$ be the regular representation of $\pi_{1}(M)$ over $\mathbf{Z}$.

We will compute the twisted Alexander invariants associated to $\rho$. By the above discussion, $A_{K}^{\rho}=H_{1}\left(B ; \mathbf{Z}[\langle s\rangle] \otimes \mathbf{Z}\left[\pi_{1}(M)\right]\right)=H_{1}(\tilde{B})$ where $\tilde{B}$ is the regular cover of $B$ associated to the homomorphism $\pi_{1}(B) \rightarrow \mathbf{Z}_{3}$ given by $x, y \mapsto 1$. Obviously $\tilde{B}$ is again a graph; $\tilde{B}$ has 3 vertices $v_{0}, v_{1}, v_{2}$ and 6 edges $x_{0}, x_{1}, x_{2}, y_{0}, y_{1}, y_{2}$ 
where $\partial x_{i}=\partial y_{i}=v_{i+1}-v_{i}$ (indices are modulo 3 ), and the covering projection $\tilde{B} \rightarrow B$ sends $x_{i}$ and $y_{i}$ to $x$ and $y$, respectively. $\pi_{1}(\tilde{B})$ can be identified with the free subgroup of $\pi_{1}(B)$ generated by $a=x y^{-1}, b=x a x^{-1}, c=x^{2} a x^{-2}$ and $d=x^{3}$, and hence $H_{1}(\tilde{B})$ is the free abelian group generated by (the homology classes of) $a, b, c$ and $d$. The action of $s$ on $H_{1}(\tilde{B})$ is easily computed by evaluating the values of $h^{2}$ on $a, b, c$ and $d$; for example, $h^{2}(a)=y^{-1} x^{-1} y^{-1} x^{-1} y=d^{-1} c a d^{-1} c^{-1} d$ in $\pi_{1}(\tilde{B}) \subset \pi_{1}(B)$, and by abelianizing, $s \cdot a=a-d$ in $H_{1}(\tilde{B})$. By computing the action on the other generators in a similar way, we obtain a matrix

$$
H=\left[\begin{array}{cccc}
1 & 0 & 0 & 1 \\
0 & 1 & 0 & 1 \\
0 & 0 & 1 & 1 \\
-1 & -1 & -1 & 2
\end{array}\right]
$$

which represents the action of $s$ on $A_{K}^{\rho}$. Thus $A_{K}^{\rho}$ is presented by $s I-H, \Delta_{K}^{\rho}(s)=$ $\operatorname{det}(s I-H)=s^{4}-s^{3}-s+1$, and $\mathcal{A}_{K}^{\rho}$ is the principal ideal generated by $\Delta_{K}^{\rho}(s)$.

\section{EXAMPLES USING ABELIAN REPRESENTATIONS}

Suppose that $K_{0}$ is a knot with a nontrivial Alexander polynomial. In this section we prove the following using abelian representations:

Theorem 4.1. There exist infinitely many knots sharing a common Seifert matrix with $K_{0}$ but having twisted Alexander polynomials of nonfibred knots.

An immediate consequence of Theorem 4.1 is that there are infinitely many knots with the same classical Alexander invariants as $K_{0}$ but twisted Alexander polynomials of nonfibred knots, as mentioned in the introduction.

We begin with a lemma, which is a consequence of well-known classical results.

Lemma 4.2. The Alexander polynomial $\Delta_{K_{0}}(t)$ of a knot $K_{0}$ is nontrivial if and only if the first homology group of the $d$-fold cyclic cover of $S^{3}$ branched along $K_{0}$ is nontrivial for some $d$.

Proof. The order of the first homology of the $d$-fold cyclic branched cover is given by the resultant

$$
R_{d}=\left|\prod_{s=0}^{d-1} \Delta_{K_{0}}\left(e^{2 \pi i s / d}\right)\right|
$$

where $R_{d}=0$ if the homology is an infinite group [5], 7], 2]. Thus if $\Delta_{K_{0}}(t)$ is trivial, then $R_{d}=1$ for all $d$.

Conversely, if $\Delta_{K_{0}}(t)$ is nontrivial, the equation $\Delta_{K_{0}}(t)=0$ has a nonzero complex root $w$. If $w$ is a $d$-th root of unity, then $R_{d}=0$. In [6], Gordon proved that if $w$ is not a root of unity, then the nonzero values of $R_{d}$ are unbounded. (Actually, more is known; Riley proved that the nonzero values of $R_{d}$ grow exponentially in $d$ [14].) This completes the proof.

By the lemma, we can choose $d$ such that the $d$-fold cyclic branched cover $M_{0}$ of the given knot $K_{0}$ has nontrivial first homology. Choose a surjection $\chi_{0}$ of $H_{1}\left(M_{0}\right)$ onto a cyclic group $\mathbf{Z}_{r}$ of order $r \geq 2$, and choose a Seifert surface $F$ of $K_{0}$. From the given data $K_{0}, \chi_{0}$, and $F$, we will construct desired knots.

We may assume that $F$ is a handlebody with one 0 -handle and $s 1$-handles, and by an isotopy we may assume that $F$ is embedded in $S^{3}$ as in Figure 1 , where $\beta$ is 
a framed $(2 s)$-string link. By a method of Akbulut and Kirby [1], $M_{0}$ is obtained by surgery on a $s(d-1)$-component link $L$ shown in Figure 2 Furthermore, a presentation of $H_{1}\left(M_{0}\right)$ is obtained as follows. Denote meridians of components of $L$ by $\gamma_{i j}(1 \leq i \leq s, 1 \leq j \leq d-1)$ as in Figure 2 , and let $A$ be the Seifert matrix of $F$ with respect to the generators of $H_{1}(F)$ represented by the 1-handles. Then $H_{1}\left(M_{0}\right)$ is generated by $\left\{\gamma_{i j}\right\}$ and

$$
\left[\begin{array}{cccc}
A+A^{T} & -A^{T} & & \\
-A & A+A^{T} & \ddots & \\
& \ddots & \ddots & -A^{T} \\
& & -A & A+A^{T}
\end{array}\right]_{s(d-1) \times s(d-1)}
$$

is a presentation matrix of $H_{1}\left(M_{0}\right)$ with respect to $\left\{\gamma_{i j}\right\}$.

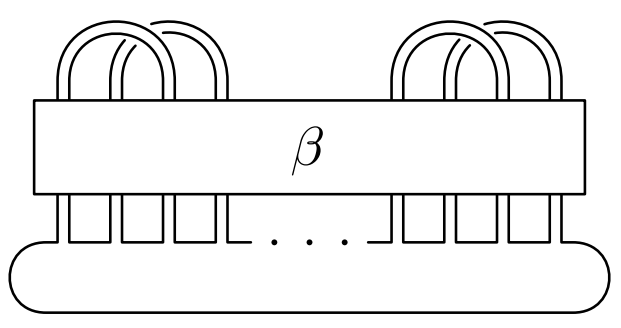

FiguRE 1.

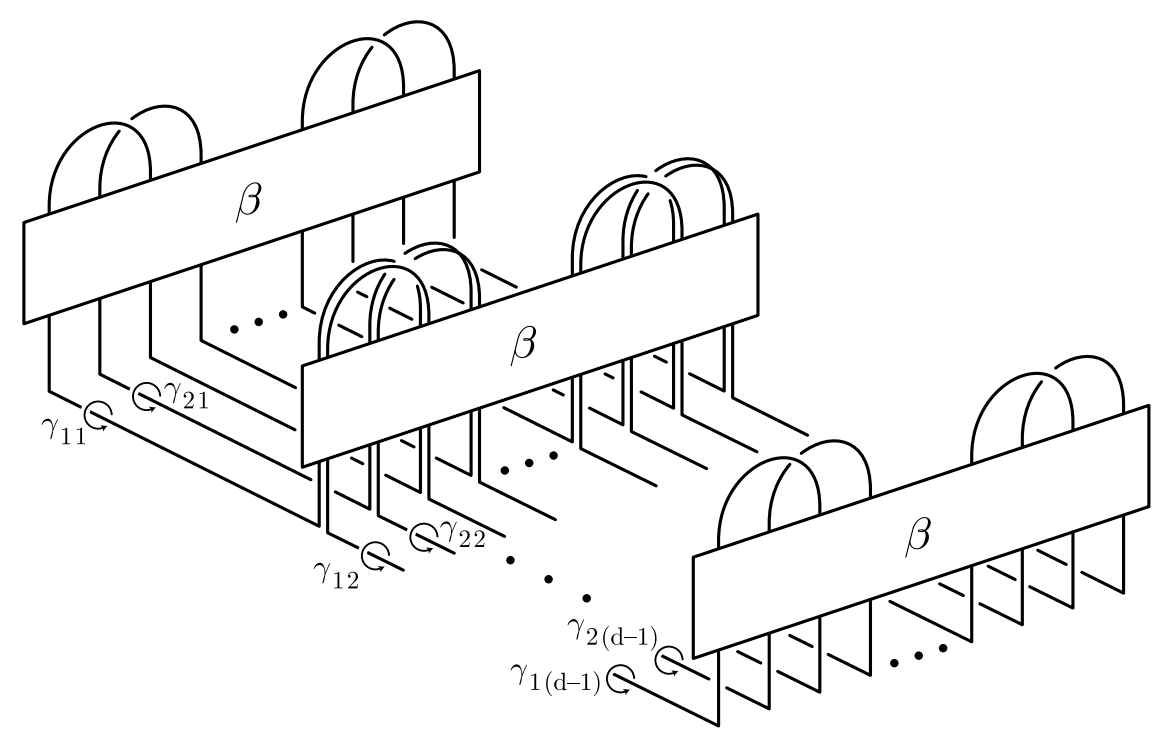

FigURE 2.

We claim that $\chi_{0}\left(\gamma_{i j}\right) \neq \chi_{0}\left(\gamma_{i(j+1)}\right)$ in $\mathbf{Z}_{r}$ for some $i$ and $j$. Suppose not. Then $\chi_{0}\left(\gamma_{i j}\right)$ is independent of $j$; let $x_{i}=\chi_{0}\left(\gamma_{i j}\right)$. Since all relations on $\gamma_{i j}$ are killed by $\chi_{0}$, we have $x\left(A+A^{T}\right)-x A=-x A^{T}+x\left(A+A^{T}\right)=0$ where $x$ is the row 
vector with entries $x_{i}$. From this we easily obtain $x\left(A-A^{T}\right)=0$. Since $A$ is a Seifert matrix of a knot, $A-A^{T}$ is nonsingular and $x=0$. This implies that $\chi_{0}$ is a trivial map, a contradiction. Thus the claim is true.

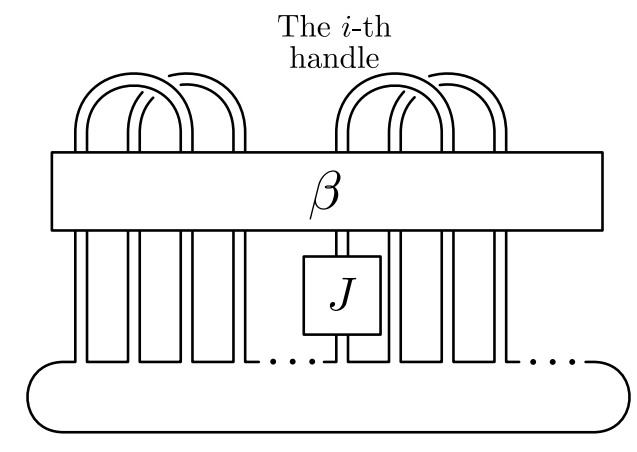

Figure 3.

Let $K$ be the knot obtained by tying a knot $J$ along the $i$-th handle of $F$ so that the Seifert form is unchanged. See Figure 3. Let $M$ be the $d$-fold cyclic branched cover of $K$. Since the tying operation does not change the Seifert matrix, the Akbulut-Kirby method gives the same presentations of $H_{1}(M)$ and $H_{1}\left(M_{0}\right)$, and hence there is a natural isomorphism between them. Let $\chi: H_{1}(M) \cong H_{1}\left(M_{0}\right) \stackrel{\chi_{0}}{\longrightarrow}$ $\mathbf{Z}_{r}$ and let

$$
\rho: \pi_{1}(M) \rightarrow H_{1}(M) \stackrel{\chi}{\rightarrow} \mathbf{Z}_{r} \rightarrow \mathrm{GL}\left(\mathbf{Z}\left[\mathbf{Z}_{r}\right]\right)
$$

where the last map is the regular representation of $\mathbf{Z}_{r}$. We will investigate the twisted Alexander invariants associated to $\rho$. Let $n$ be the order of $\chi_{0}\left(\gamma_{i j}\right)-$ $\chi_{0}\left(\gamma_{i(j+1)}\right)$ in $\mathbf{Z}_{r}$. Note that $n \geq 2$. Let $M_{J}$ be the $n$-fold cyclic cover of $S^{3}$ branched along $J$. Then

Theorem 4.3. If $H_{1}\left(M_{J}\right)$ is nontrivial, then the twisted Alexander invariant associated to $\rho$ does not satisfy the conclusions of Theorem 1.1.

Actually, there exist infinitely many knots $J$ satisfying the hypothesis of Theorem 4.3. For example, the following lemma says that the hypothesis is true for any knot $J$ having the same Seifert matrix as that of the figure eight knot.

Lemma 4.4. If $\left[\begin{array}{cc}1 & 1 \\ 0 & -1\end{array}\right]$ is a Seifert matrix of $J$, then the first homology of the $n$-fold cyclic cover of $S^{3}$ branched along $J$ is nontrivial for all $n \geq 2$.

Proof. Since $A$ is nonsingular, $H^{n}-I$ is a presentation matrix of the first homology of the $n$-fold cyclic branched cover of $J$, where $H=A^{-1} A^{T}=\left[\begin{array}{cc}2 & -1 \\ -1 & 1\end{array}\right]$. It suffices to show that $H^{n}-I$ is not unimodular for $n \geq 2$. Let $H^{n}=\left[\begin{array}{ll}a_{n} & b_{n} \\ b_{n} & c_{n}\end{array}\right]$. (Note that $H$ is a symmetric matrix.) Then it is easily shown that $a_{n} \geq 5, b_{n} \leq-3$ and $c_{n} \geq 2$ for $n \geq 2$, by an induction. Since $\operatorname{det}(H)=1$, we have $\operatorname{det}\left(H^{n}-I\right)=\left(a_{n}-1\right)\left(c_{n}-1\right)-b_{n}^{2}=\operatorname{det}\left(H^{n}\right)+1-a_{n}-c_{n}=2-a_{n}-c_{n} \leq-5$.

Since a different choice of $J$ produces a different knot $K$ (e.g., by the uniqueness of the torus decomposition of knot complements), Theorem 4.1 follows Theorem 4.3 
Proof of Theorem 4.3. Let $\Lambda=\mathbf{Z}[\langle s\rangle]$ and $N$ be the $d$-fold cyclic cover of the exterior of $K$ as before. By definition, $A_{K}^{\rho}$ is equal to the twisted homology $H_{1}(N ; \mathcal{V})$ where $\mathcal{V}=\Lambda \otimes \mathbf{Z} \mathbf{Z}\left[\mathbf{Z}_{r}\right]=\mathbf{Z}\left[\langle s\rangle \oplus \mathbf{Z}_{r}\right]$.

Let $U$ be an unknotted solid torus in $S^{3}-F$ which links the $i$-th handle of $F$. The exterior of $K$ is obtained from the exterior of $K_{0}$ by removing the interior of $U$ and by replacing it with the exterior of $J$ along the boundary. The meridian (resp. the longitude) of $J$ is identified with a curve on $\partial U$ which is homotopic to the core of $U$ (resp. null-homotopic in $U$ ). Since the linking number of $U$ and $K_{0}$ is zero, $U$ is lifted to the $d$-fold cyclic cover $N_{0}$ of the exterior of $K_{0} . N$ is obtained by removing the interiors of all lifts of $U$ from $N_{0}$ and filling $d$ copies of the exterior of $J$ along the boundaries. Viewing $N_{0}$ as a subspace of $M_{0}, \gamma_{i j}-\gamma_{i(j+1)}$ is homologous to the core of a lift of $U$ in $M_{0}$. In $N$, the boundary of that lift bounds a copy of the exterior of $J$. Denote it by $E_{J}$.

Let $Y$ be the closure of $N-E_{J}$. Applying the Mayer-Vietoris theorem to $N=$ $E_{J} \cup Y$, we obtain an exact sequence

$$
\begin{aligned}
\quad \cdots \rightarrow H_{1}\left(\partial E_{J} ; \mathcal{V}\right) \rightarrow H_{1}\left(E_{J} ; \mathcal{V}\right) \oplus H_{1}(Y ; \mathcal{V}) \rightarrow H_{1}(N ; \mathcal{V}) & \rightarrow H_{0}\left(\partial E_{J} ; \mathcal{V}\right) \rightarrow H_{0}\left(E_{J} ; \mathcal{V}\right) \oplus H_{0}\left(E_{J} ; \mathcal{V}\right) .
\end{aligned}
$$

The twisted homologies of $E_{J}$ and $\partial E_{J}$ have a simple structure as follows. First we observe that (1) the linking number of $U$ and $K_{0}$ is zero, and (2) the map $\chi$ sends the meridian of $J$ in $E_{J}$ to the element $\chi_{0}\left(\gamma_{i j}-\gamma_{i(j+1)}\right)$, which is of order $n$ in $\mathbf{Z}_{r}$. From the observations, the $\left(\langle s\rangle \oplus \mathbf{Z}_{r}\right)$-covering $\widetilde{E}_{J}$ of $E_{J}$ is a union of infinitely many copies of the $n$-fold cyclic cover $N_{J}$ of $E_{J}$ and we have $H_{*}\left(E_{J} ; \mathcal{V}\right)=H_{*}\left(\mathcal{V} \otimes_{\mathbf{z}\left[\langle s\rangle \oplus \mathbf{Z}_{r}\right]}\right.$ $\left.C_{*}\left(\widetilde{E}_{J}\right)\right)=H_{*}\left(\widetilde{E}_{J}\right)=H_{*}\left(N_{J}\right)^{r / n} \otimes_{\mathbf{z}} \Lambda$. Similarly, $H_{*}\left(\partial E_{J} ; \mathcal{V}\right)=H_{*}\left(\partial N_{J}\right)^{r / n} \otimes_{\mathbf{z}} \Lambda$. Therefore, $H_{0}\left(\partial E_{J} ; \mathcal{V}\right) \rightarrow H_{0}\left(E_{J} ; \mathcal{V}\right)$ is an isomorphism, and Coker $\left\{H_{1}\left(\partial E_{J} ; \mathcal{V}\right) \rightarrow\right.$ $\left.H_{1}\left(E_{J} ; \mathcal{V}\right)\right\}$ is isomorphic to $\operatorname{Coker}\left\{H_{1}\left(\partial N_{J}\right) \rightarrow H_{1}\left(N_{J}\right)\right\}^{r / n} \otimes_{\mathbf{Z}} \Lambda=H_{1}\left(M_{J}\right)^{r / n} \otimes_{\mathbf{Z}}$ $\Lambda$. By the lemma below, an annihilator of $H_{1}(N ; \mathcal{V})$ annihilates $H_{1}\left(M_{J}\right) \otimes_{\mathbf{Z}} \Lambda$ as well.

Lemma 4.5. Suppose that $A$ and $B$ are modules and $C$ is a submodule of $A \oplus B$. Let $p: A \oplus B \rightarrow A$ be the canonical projection. Then an annihilator of $(A \oplus B) / C$ annihilates $A / p(C)$ as well.

Proof. Viewing $A$ and $B$ as submodules of $A \oplus B$,

$$
(A \oplus B) / C=((A+C)+(B+C)) / C=(A+C) / C+(B+C) / C .
$$

Thus $(A+C) / A \cong A / A \cap C$ is a submodule of $(A \oplus B) / C$. Since $p(C)$ contains $A \cap C, A / p(C)$ is a quotient of $A /(A \cap C)$. The conclusion follows.

Since $H_{1}\left(M_{J}\right)$ is nontrivial, $H_{1}\left(M_{J}\right) \otimes \Lambda$ is never annihilated by any nonzero monic polynomial, and hence so is $A_{K}^{\rho}$. By Corollary 3.3, $A_{K}^{\rho}$ is not a twisted Alexander module of a fibred knot.

Remark 4.6. If one were interested in fibredness only, regardless of the relationship with the twisted Alexander invariants, then it could be shown that our example $K$ is not fibred by a straightforward argument. In general, if a knot $K$ has a nontrivial companion $J$ with zero winding number, $K$ is not fibred. For, $\pi_{1}\left(E_{J}\right) \rightarrow \pi_{1}\left(E_{K}\right)$ is an injection, and since the winding number is zero, the image is contained in the commutator subgroup $\left[\pi_{1}\left(E_{K}\right), \pi_{1}\left(E_{K}\right)\right]$. Since $\pi_{1}\left(E_{J}\right)$ is not 
free, $\left[\pi_{1}\left(E_{K}\right), \pi_{1}\left(E_{K}\right)\right]$ is also not free. This shows that $K$ is not fibred. The author thanks an anonymous referee for pointing this out.

Remark 4.7. Our construction is similar to one in [9], which was used to illustrate that the twisted Alexander module of the complement of a knot is not necessarily $\Lambda$-torsion. The above argument shows that an analogous result holds for the twisted Alexander module of a branched cover of a knot as well; if $H_{1}\left(M_{J}\right)$ is free abelian, $A_{K}^{\rho}$ is not $\Lambda$-torsion, since any annihilator of $A_{K}^{\rho}$ annihilates $\Lambda$ and hence must be zero by the proof of Theorem 4.3. This is a significant difference between the twisted Alexander invariants and the classical Alexander invariants.

Remark 4.8. If a knot $K$ has a trivial Alexander polynomial, twisted Alexander invariants associated to abelian representations are no more than the classical ones; indeed all abelian representations are trivial since the first homologies of cyclic branched covers of $K$ always vanish by Lemma 4.2 However, invariants associated to nonabelian representations are still interesting, as shown in the next section.

\section{EXAMPLES USING NONABELIAN REPRESENTATIONS}

In this section we illustrate examples of knots that have trivial Alexander polynomials but do not have twisted Alexander modules of fibred knots using nonabelian representations.

For a knot $J$, consider the link shown in Figure 4 Denote its components by $L_{1}$ and $L_{2}$ as in the figure. As usual, $J$ is tied so that the writhe of the diagram is unchanged. Performing (1/1)-surgery along $L_{2}$, the ambient space still remains $S^{3}$ but the other component $L_{1}$ becomes a knotted circle $K$.

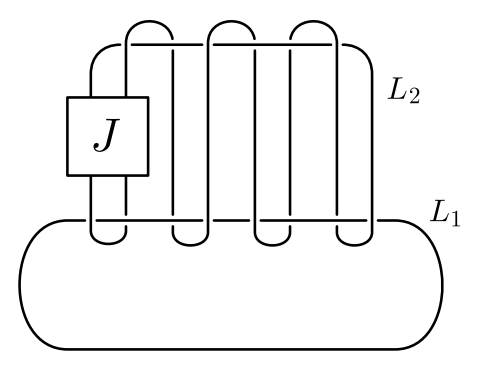

Figure 4.

We remark that a similar construction was used to produce a knot with a given Alexander polynomial in [12] and [15, 7.C.5]. Actually, by the same arguments, it is easily seen that for any $J$ our construction produces a knot $K$ with a trivial Alexander polynomial.

Let $M_{J}$ be the 3 -fold cyclic cover of $S^{3}$ branched along $J$. Our goal is to prove

Theorem 5.1. If $H_{1}\left(M_{J}\right)$ is nontrivial, $K$ does not have twisted Alexander invariants of fibred knots.

Proof. First we consider a special case where $J$ is unknotted. Let $K_{0}$ be the knot obtained from an unknot $J$ by the above construction. Let $M_{0}$ be the double branched cover of $K_{0}$. Cutting $S^{3}$ along the obvious disk bounded by $L_{1}$ and pasting two copies, we obtain a surgery diagram of $M_{0}$ with two components, which is shown in Figure 5 


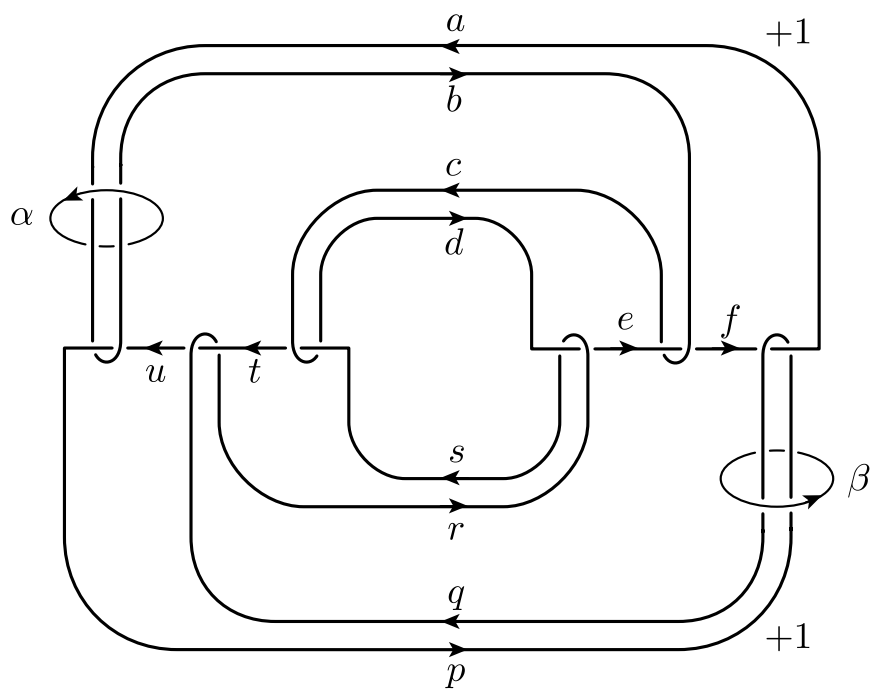

FiguRE 5 .

The Wirtinger presentation of the diagram in Figure 5 (forgetting the framing) is as follows: there are 12 generators $a, b, c, d, e, f, p, q, r, s, t, u$, and 12 relations

$$
\begin{aligned}
& a=q^{-1} f q, \quad b=p^{-1} a p, \quad c=e^{-1} b e, \quad d=s c s^{-1}, \quad e=r d r^{-1}, \quad f=b^{-1} e b, \\
& p=b^{-1} u b, \quad q=a^{-1} p a, \quad r=t^{-1} q t, \quad s=d r d^{-1}, \quad t=c s c^{-1}, \quad u=q^{-1} t q
\end{aligned}
$$

where any one of the relations is redundant. Adding the relations

$$
\text { qpes }{ }^{-1} r^{-1} b f^{-1}=1, \quad b a t d^{-1} c^{-1} q u^{-1}=1
$$

which represent the effect of surgery, we obtain a presentation of $\pi_{1}\left(M_{0}\right)$.

We define a homomorphism $\phi_{0}$ of $\pi_{1}\left(M_{0}\right)$ into $A_{5}$, the group of even permutations on $\{1, \ldots, 5\}$, by assigning values to generators as follows. (Cycle notations are used to represent elements of $A_{5}$.)

$$
\begin{aligned}
& \phi_{0}(a)=(132), \quad \phi_{0}(b)=(142), \quad \phi_{0}(c)=(125), \\
& \phi_{0}(d)=(243), \quad \phi_{0}(e)=(145), \quad \phi_{0}(f)=(152), \\
& \phi_{0}(p)=(13542), \quad \phi_{0}(q)=(15432), \quad \phi_{0}(r)=(12534), \\
& \phi_{0}(s)=(14523), \quad \phi_{0}(t)=(15324), \quad \phi_{0}(u)=(14352) \text {. }
\end{aligned}
$$

It is tedious but straightforward to verify that all relations are killed by $\phi_{0}$, and in addition, $\phi_{0}$ is a surjection.

A representation for the general case is induced by the homomorphism $\phi_{0}$ as follows. Let $K$ be the knot obtained from a knot $J$ (not necessarily unknotted) by the above construction. Let $U$ and $V$ be tubular neighborhoods of the curves $\alpha$ and $\beta$ shown in Figure 5 Then the double branched cover $M$ of $K$ is obtained from $M_{0}$ by removing the interiors of $U$ and $V$ and by filling two copies $E_{J}$ and $E_{J}^{\prime}$ of the exterior of $J$ along $\partial U$ and $\partial V$, respectively. By the Seifert-van Kampen theorem, $\pi_{1}(M)$ is an amalgamated product of $\pi_{1}\left(E_{J}\right), \pi_{1}\left(E_{J}^{\prime}\right)$ and $\pi_{1}\left(M-\left(E_{J} \cup E_{J}^{\prime}\right)\right)$. Let $\phi_{1}$ and $\phi_{2}$ be homomorphisms of $H_{1}\left(E_{J}\right)$ and $H_{1}\left(E_{J}^{\prime}\right)$ into $A_{5}$ which send the meridian to (243) and (253), respectively. Since $\phi_{0}(\alpha)=\phi_{0}\left(a b^{-1}\right)=(243)$ and 
$\phi_{0}(\beta)=\phi_{0}\left(p q^{-1}\right)=(253)$, the homomorphisms

$$
\begin{gathered}
\pi_{1}\left(M-\left(E_{J} \cup E_{J}^{\prime}\right)\right) \rightarrow \pi_{1}\left(M_{0}\right) \stackrel{\phi_{0}}{\longrightarrow} A_{5}, \\
\pi_{1}\left(E_{J}\right) \rightarrow H_{1}\left(E_{J}\right) \stackrel{\phi_{1}}{\longrightarrow} A_{5}, \\
\pi_{1}\left(E_{J}^{\prime}\right) \rightarrow H_{1}\left(E_{J}^{\prime}\right) \stackrel{\phi_{2}}{\longrightarrow} A_{5}
\end{gathered}
$$

induce a homomorphism $\phi: \pi_{1}(M) \rightarrow A_{5}$. Let $\rho$ be the representation of $\pi_{1}(M)$ obtained by composing $\phi$ with the regular representation of $A_{5}$.

Now we are ready to apply the arguments of the previous section. Since $\phi$ sends the meridian of $J$ in $E_{J}$ to an element (243), that is of order 3 in $A_{5}$, an annihilator of $A_{K}^{\rho}$ annihilates $H_{1}\left(M_{J}\right) \otimes \mathbf{z} \Lambda$ as in the proof of Theorem 4.3. If $H_{1}\left(M_{J}\right)$ is nontrivial, $H_{1}\left(M_{J}\right) \otimes_{\mathbf{z}} \Lambda$ is never annihilated by any monic polynomial, and so is $A_{K}^{\rho}$. Therefore, $K$ does not have twisted Alexander invariants of fibred knots by Corollary 3.3 .

Remark 5.2. As done with the examples in the previous section, the arguments of Remark 4.6 can also be used to show nonfibredness.

Remark 5.3. By Lemma 4.4, there are infinitely many knots $J$ satisfying the hypothesis of Theorem 5.1 and hence by the above construction we can produce infinitely many knots $K$ with the desired property.

\section{ACKNOWLEDGEMENTS}

The author thanks an anonymous referee for comments on several crucial points. Without his/her help, this paper would not have been improved to the present form.

This work was partially supported by the Post-doctoral Fellowship Program of Korea Science and Engineering Foundation (KOSEF).

\section{REFERENCES}

[1] S. Akbulut and R. Kirby, Branched covers of surfaces in 4-manifolds, Math. Ann. 252 (1979/80), no. 2, 111-131. MR 82j:57001

[2] G. Burde and H. Zieschang, Knots, Walter de Gruyter \& Co., Berlin, 1985. MR 87b:57004

[3] T. D. Cochran, Noncommutative Knot Theory, arXiv:math.GT/0206258.

[4] R. H. Crowell and R. H. Fox, Introduction to knot theory, Springer-Verlag, New York, 1977, Reprint of the 1963 original, Graduate Texts in Mathematics, No. 57. MR 56:3829

[5] R. H. Fox, Free differential calculus. III. Subgroups, Ann. of Math. (2) 64 (1956), 407-419. MR 20:2374

[6] C. Gordon, Knots whose branched cyclic coverings have periodic homology, Trans. Amer. Math. Soc. 168 (1972), 357-370. MR 45:4394

[7] - Some aspects of classical knot theory, Knot theory (Proc. Sem., Plans-sur-Bex, 1977), Springer, Berlin, 1978, pp. 1-60. MR 80f:57002

[8] B. J. Jiang and S. C. Wang, Twisted topological invariants associated with representations, Topics in knot theory (Erzurum, 1992), Kluwer Acad. Publ., Dordrecht, 1993, pp. 211-227.

[9] P. Kirk and C. Livingston, Twisted Alexander invariants, Reidemeister torsion, and CassonGordon invariants, Topology 38 (1999), no. 3, 635-661. MR 2000c:57010

[10] Twisted knot polynomials: inversion, mutation and concordance, Topology $\mathbf{3 8}$ (1999), no. 3, 663-671. MR 2000c:57011

[11] T. Kitano, Twisted Alexander polynomial and Reidemeister torsion, Pacific J. Math. 174 (1996), no. 2, 431-442. MR 97g:57007

[12] J. P. Levine, A characterization of knot polynomials, Topology 4 (1965), 135-141. MR 31:5194

[13] X.-S. Lin, Representations of knot groups and twisted Alexander polynomials, Acta Math. Sinica (Engl. Ser). 17 (2001), 361-380. MR 2003f:57018 
[14] R. Riley, Growth of order of homology of cyclic branched covers of knots, Bull. London Math. Soc. 22 (1990), no. 3, 287-297. MR 92g:57017

[15] D. Rolfsen, Knots and links, Publish or Perish Inc., Berkeley, CA, 1976, Mathematics Lecture Series, No. 7; corrected reprint, 1990. MR 58:24236, MR 95c:57018

[16] M. Wada, Twisted Alexander polynomial for finitely presentable groups, Topology 33 (1994), no. 2, 241-256. MR 95g:57021

Department of Mathematics, Indiana University, Bloomington, Indiana 47405

E-mail address: jccha@indiana.edu

Current address: Information and Communication University, 119 Munjiro, Yuseong-gu, Daejeon 305-714, Korea

E-mail address: jccha@icu.ac.kr 